\title{
Non-linear Predictive Control for a Distillation Column
}

\author{
A. Rueda*, S. Cristea ${ }^{*}$, C. de Prada ${ }^{*}$ R. De Keyser ${ }^{* *}$ \\ "Department of Systems Engineering and Automatic Control \\ Faculty of Sciences, c/ Real de Burgos, s/n, University of Valladolid, Spain \\ fax: +34983423161; tel: +34983423164 \\ prada@autom.uva.es,smaranda@autom.uva.es,almudena@cta.uva.es \\ **ESA-Department of Electrical Energy, Systems \& Automation \\ Ghent University, Technologiepark-Zwijnaarde 913, 9052 GENT, Belgium \\ rdk@autoctrl.Ugent.be
}

\begin{abstract}
In this paper an alternative approach to non-linear predictive control is presented. It is based on iterative linearisation of the model response so that the same closed loop responses as in the pure non-linear approach are obtained but with reduced computation times and more efficient optimisation tools. The method is applied to a high purity distillation column and some results are presented showing the behaviour of the proposed algorithm.
\end{abstract}

Keywords: non-linear predictive control, distillation columns, constrained systems

\section{INTRODUCTION}

MPC is now recognised in the industrial world as a proven technology, capable of dealing with a wide range of multivariable constrained control problems. Nevertheless, most of the industrial controllers are based on linear internal models which limits its applicability. Because of it, nonlinear model predictive control NMPC has received a lot of attention in the latest years, both from the point of view of its properties (Camacho et al. 2005) and implementation (Bartusiak, 2005). Referring to this last aspect, the main drawback is the computational burden that NMPC implies.

While linear MPC with constraints can solve the associated optimisation problem each sampling time using QP or LP algorithms, for which very efficient codes are available, NMPC relies on non-linear programming (NLP) methods such as SQP, that are known to be far more CPU demanding. Several schemes have been proposed to deal with this problem among them the well known sequential and simultaneous approaches.
For sequential solutions, the model is solved by integration at each iteration of the optimisation routine. Only the control parameters remain as degrees of freedom in the NLP. Simulation and optimisation calculations are performed sequentially, one after the other. The approach can easily be coupled with advanced simulation tools. In contrast, simultaneous model solution and optimisation includes both the discretized model states and controls as decision variables and the model equations are appended to the optimisation problem as equality constraints. This can greatly increase the size of the optimisation problem. Most of the times SQP like algorithms are used. In spite of other recent proposals (Bock, 2005) computation time remains a difficulty in order to implement NMPC in real processes .

In order to overcome this drawback several alternatives to standard NMPC have been proposed like NMPC techniques based on model linearization. An overview of these can be found in (Bequette, 1991; Henson, 1998; Morari and Lee, 1999). Another approach (De Keyser, 1998) is considered in this paper, which uses a local linearization of the process at each sampling time to compute an 'optimized response', analogous to the 'forced response' of linear MPC methods, and the procedure is applied iteratively until the same nonlinear solution is reached using only QP algorithms that are both fast and reliable.

This paper describes the iterative linearization technique and compares a NMPC algorithm using the sequential approach and a version of the iterative linearization applied to a nontrivial process control example. Section 2 presents the process, an industrial distillation column of an alcohol plant, and its control objectives. Section 3 deals with the predictive controller algorithm, while section 4 shows several results. The paper ends with some conclusions. 


\section{PROCESS DESCRIPTION}

\subsection{Distillation column}

Distillation is an important process widely used in industry. In our case we focus the attention to a high purity distillation being a multi-component one, deals with azeotropes, which makes it more complex.

The regulation goal is keeping the ethanol molar concentration of the column-bottom and the water molar concentration of the column-top close to its set points. To obtain this control objective two manipulated variables were selected: the lateral neutral alcohol flow and the steam inflow to the reboiler. Other variables are kept under control by local PID controllers: the level in the column bottom by manipulating the outflow, the accumulator level by manipulating the reflux and the head column pressure by manipulating the refrigerant flow to the condenser. Flows of steam to the reboiler and neutral alcohol are maintained using PID controllers.

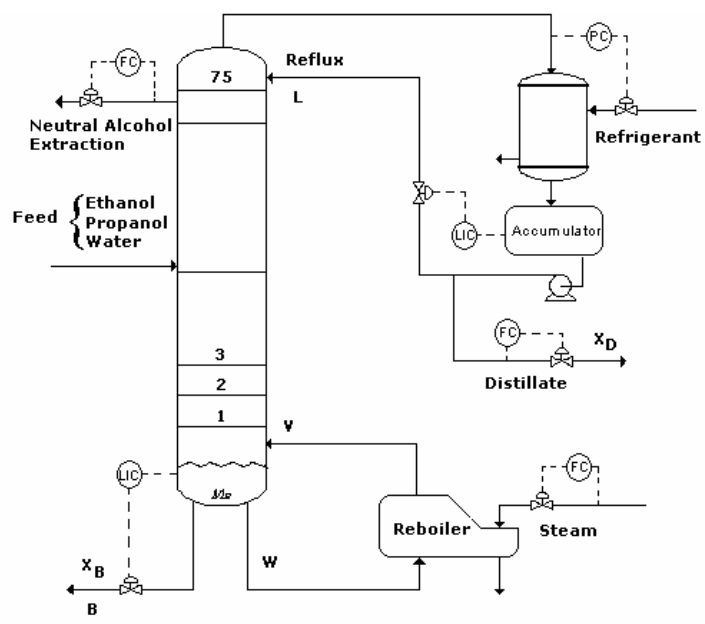

Fig. 1: Simplified sketch of the Neutral Alcohol Distillation Column

\subsection{Plant modeling}

A detailed model of the process has been developed and tested with real data. The main hypothesis considered are listed next:

- A non-constant vapour flow is considered, and it is calculated on the basis of the energy balance assumed for each of the plates.

- Total pressure loss of the column is distributed linearly among all the plates.

- The vapour and liquid that flow from the plate are in a state of thermal equilibrium and also at the same pressure.

- The vapour and liquid that flow from the plate are not in phase equilibrium owing to the definition of a Murphree efficiency.

- The vapour-liquid equilibrium is represented taking into consideration:

- Vapour phase as being ideal column of the sugar industry. The feed is a mix of three main products: water, ethanol and propanol, and the ethanol is extracted from the accumulator and, mainly, using a top lateral extraction. The column has 75 plates and besides

- Liquid phase as being not ideal: Wilson's model is employed to calculate the activity coefficient

The equations that represent the behaviour of a generic plate are the same for the complete column (all the variables are described in the attached nomenclature):

- Overall balance of material:

$$
\frac{d M_{n}}{d t}=L_{n+1}+V_{n-1}+F_{n}-L_{n}-V_{n}-S_{n}
$$

- Overall balance at component $j$ :

$$
\begin{aligned}
& \frac{d\left(M_{n} x_{n}^{j}\right)}{d t}=L_{n+1} x_{n+1}^{j}+V_{n-1} y_{n-1}^{j}+F_{n} z_{n}^{j}-L_{n} x_{n}^{j}-V_{n} y_{n}^{j}-S_{n} x_{n}^{j} \\
& \sum_{j} x_{n}^{j}=1
\end{aligned}
$$

- Overall energy balance:

The changes in the specific enthalpy of the liquid phase are generally very small compared with the total enthalpy of the plate. This means that, normally, the energy balance can be reduced to an algebraic equation which is used as the basis to calculate the flow of vapour from the plate.

$$
L_{n+1} h_{n+1}+V_{n-1} H_{n-1}+F_{n} h_{f, n}-L_{n} h_{n}-V_{n} H_{n}-S_{n} h_{n}=0
$$

- Temperature calculation:

The bubble temperature is that one which is in equilibrium with a known composition of the liquid at a determined pressure which is also known.

$$
\sum_{j} y_{n, j}=1
$$

Equilibrium ratio between vapour-liquid phases:

$$
y_{n, j}{ }^{*}=\frac{\gamma_{n}^{j} P_{n, j}^{s a t}}{P_{n}} x_{n}^{j}
$$

- Total pressure on plate $n$ :

$$
\begin{aligned}
& \mathrm{P}_{\mathrm{n}}=\mathrm{P}_{\mathrm{n}+1}+\Delta \mathrm{P}_{\mathrm{n}} \\
& \Delta \mathrm{P}_{\mathrm{n}}=\left(\frac{\mathrm{V}_{0}}{\mathrm{~K}}\right)^{2}
\end{aligned}
$$

- Flow of liquid from plate $n$ :

The flow of liquid is calculated on the basis of Francis' formula for segmented sinks:

$$
\begin{aligned}
h_{o w} & =664 \cdot\left(\frac{Q}{L_{w}}\right)^{2 / 3} \\
Q_{n} & =1.8482 L_{w}\left(h_{o w, n}\right)^{1.5}
\end{aligned}
$$

The model involves 2700 equations and 157 states and was simulated and tested using the EcosimPro modelling and simulation language. 


\section{PREDICTIVE CONTROLLER}

Nonlinear predictive control (NMPC) is a natural extension of the linear MPC technique. The algorithm is also based on the use of an internal plant model, which captures the main process characteristics. As mentioned in the introduction, two different formulations of NMPC were compared, a direct NMPC and a iterative linearization technique.

\subsection{NMPC Controller}

The objective of the non-linear model predictive control (NMPC) is finding the future optimal manipulated variable sequence in order to minimize a function based on a desired output trajectory over a prediction horizon. The cost function is the integral over the squares of the residuals between the model predicted outputs $y_{\text {pred }}$ and the set point values $r$ over the prediction time $N_{2} \tau$ (where $N_{2}$ is the prediction horizon and $\tau$ is the sampling time). A typical formulation is

$$
\begin{aligned}
\min _{u(t), \ldots, u\left(t+N_{u}-1\right)} J= & \sum_{j=N_{1}}^{N_{2}}\left(y_{\text {pred }}(t+j)-r(t+j)\right)^{2}+ \\
& \sum_{k=0}^{N_{u}-1} \beta[\Delta u(t+j)]^{2}
\end{aligned}
$$

The change in the manipulated variable $u$ is also included in the minimization. The minimization (9) is done subject to the continuous model equations and to the typical restrictions applied on the manipulated and controlled variables:

$$
\begin{array}{lc}
u_{\text {min }} \leq u(t+k) \leq u_{\max } & k=0, N_{2} \\
\Delta u_{\text {min }} \leq \Delta u(t+k) \leq \Delta u_{\text {max }} & k=0, N u-1 \\
y_{\text {min }} \leq y(t+k) \leq y_{\text {max }} & k=1, N_{2}
\end{array}
$$

Of the $N_{u}$ moves in the optimal control sequence, only the first component is implemented.

Within this schema the continuous formulation of the process model is used to calculate the predictions $y_{\text {pred }}(t)$ needed for the minimization of (9) using a dynamic simulator (Fig. 2).

In this formulation, the model equations are not explicit restrictions to the optimisation problem, being the manipulated variables the only decision variables. The simulation package will integrate the model equations along the prediction horizon taking as initial conditions the current process state and evaluating the formulated objective at the end of the integration. Path constraints are implemented as penalization functions when a constraint is violated in the simulation block.

The controller law solution leads to a non-linear programming problem, which could be formulated generically as a real time minimization of a non-linear function subject to constraints.

\subsection{Nonlinear iterative EPSAC formulation}

The key idea of this formulation is to approximate the nonlinear predictions by iterative linearizations around future trajectories, so that they converge to the same non-linear optimal solution. For this purpose, the future sequence of manipulated variables is considered as the sum of a basic future control scenario, called $u_{\text {base }}(t+k / t), k \geq 0$ and optimizing future control actions $\delta \mathrm{u}(\mathrm{t}+\mathrm{k} / \mathrm{t}), 0 \leq \mathrm{k} \leq \mathrm{N}_{\mathrm{u}}-1$ :

$$
u(t+k / t)=u_{\text {base }}(t+k / t)+\delta u(t+k / t)
$$

In this way the output predictions can be considered as being the cumulative results of two effects:

$$
\mathrm{y}(\mathrm{t}+\mathrm{k} / \mathrm{t}) \approx \mathrm{y}_{\text {base }}(\mathrm{t}+\mathrm{k} / \mathrm{t})+\mathrm{y}_{\text {optimize }}(\mathrm{t}+\mathrm{k} / \mathrm{t})
$$

The component $y_{\text {base }}(t+k / t)$ is calculated using the nonlinear model and the known (postulated) sequence $u_{\text {base }}(t+k / t)$ as the model input. The other component $y_{\text {optimize }}(t+k / t)$ is the cumulative effect of a series of impulse inputs and a step input (De Keyser, 1998).

$$
\begin{aligned}
y_{\text {oppiniziee }}(t+k / t)= & h_{k} \delta u(t / t)+h_{k-1} \delta u(t+1 / t) \\
& +\ldots+g_{k-N_{u}+1} \delta u\left(t+N_{u}-1 / t\right)
\end{aligned}
$$

Using matrix notation, the prediction equation becomes

$$
\mathbf{Y}=\overline{\mathbf{Y}}+\mathbf{G U}
$$

where

$$
\begin{aligned}
\mathrm{Y} & =\left[\begin{array}{llll}
y\left(t+N_{1} / t\right) & \ldots & y\left(t+N_{2} / t\right)
\end{array}\right]^{T} \\
\overline{\mathrm{Y}} & =\left[\begin{array}{lllll}
y_{\text {base }}\left(t+N_{1} / t\right) & \ldots & y_{\text {base }}\left(t+N_{2} / t\right)
\end{array}\right]^{T} \\
\mathrm{U} & =\left[\begin{array}{lllll}
\delta u(t / t) & \ldots & \delta u\left(t+N_{u}-1 / t\right)
\end{array}\right]^{T} \\
\mathrm{G} & =\left[\begin{array}{lllll}
h_{N_{1}} & h_{N_{1}-1} & h_{N_{1}-2} & \ldots & g_{N_{1}-N_{u}+1} \\
h_{N_{1}+1} & h_{N_{1}} & h_{N_{1}-1} & \ldots & g_{N_{1}-N_{u}+2} \\
\ldots & \ldots & \ldots & \ldots & \ldots \\
h_{N 2} & h_{N_{2}-1} & h_{N_{2}-2} & \ldots & g_{N_{2}-N_{u}+1}
\end{array}\right]
\end{aligned}
$$

where the parameters $h_{1}, h_{2}, \ldots, h_{k}, \ldots h_{N_{2}}$ are the coefficients of the unit impulse response of the system at the current operating point, whereas the values $g_{k}$ refer to the unit step response coefficients.

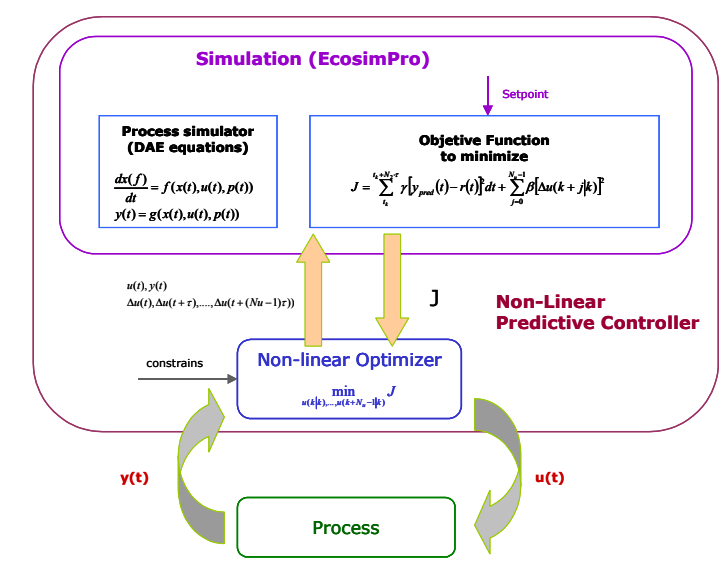

Fig. 2 - Non-linear Controller - Continuous implementation Framework. 
For a complex dynamic model obtaining a linearized model is not an easy task. To avoid it, a possible alternative is to use the non-linear model to calculate the coefficients $h_{k}$ and $g_{k}$ through the model simulation procedure. Recalling that $h_{k}=g_{k}-g_{k-1}$, a new formula is obtained for $y_{\text {optimize }}(t+k / t)$ :

$$
\begin{aligned}
& y_{\text {optimize }}(t+k / t)=g_{k}\left[u(t-1)+\Delta u(t)-u_{\text {base }}(t)\right] \\
& +\sum_{i=1}^{N_{u}-1} g_{k-i}\left[\Delta u(t+i)+u_{\text {base }}(t+i-1)-u_{\text {base }}(t+i)\right]
\end{aligned}
$$

The step response coefficients can be calculated each sample instant simulating the non-linear model of the system with a particular future sequence $\mathrm{u}^{*}(\mathrm{t}+\mathrm{k} / \mathrm{t})$ taking as initial conditions the current process state and evaluating the predictions $y^{*}(t+k / t)$.

Based on (14)...(16) the cost function is a quadratic form in U

$$
\begin{aligned}
J & =\gamma \sum_{k=N_{1}}^{N_{2}}[y(t+k / t)-r(t+k / t)]^{2}+\beta \sum_{k=0}^{N_{u}-1}[\Delta u(t+k / t)]^{2}= \\
& =\gamma(\mathbf{R}-\overline{\mathbf{Y}}-\mathbf{G U})^{T}(\mathbf{R}-\overline{\mathbf{Y}}-\mathbf{G U})+\beta(\mathbf{A U}+\mathbf{b})^{T}(\mathbf{A U}+\mathbf{b})
\end{aligned}
$$

and the optimisation problem, the minimization of $J$ subject to the constraints (10), is solved with simple quadratic programming techniques (QP).

The calculation of the predicted output (12) involves the superposition principle. When a nonlinear system model is used, above strategy is only valid - from a practical point of view - if the term $y_{\text {optimize }}(t+k \mid t)$ is small enough compared to the term $y_{\text {base }}(t+k \mid t)$ (when this term would be zero, the superposition principle would no longer be involved). The term $y_{\text {optimize }}(t+k \mid t)$ will be 'small' if $\delta u(t+k \mid t)$ is small (13). Referring to eq. (11), $\delta u(t+k \mid t)$ will be small if $u_{\text {base }}(t+k \mid t)$ is chosen 'close' to the optimal $u(t+k \mid t)$.

This can be realized iteratively, by executing the following steps at each controller sampling instant:

1. Initialize $u_{\text {base }}(t+k \mid t)$ as:

$u_{\text {base }}^{1}(t+k \mid t)=u^{*}(t+k / t-1)$, i.e. the optimal control sequence as computed during the previous sampling instant; in other words: $u^{*}(t+k / t-1)$ is used as a $1^{\text {st }}$ estimate for $u^{*}(t+k / t)$

2. Calculate $\delta u^{1}(t+k \mid t)$ by minimizing eq. (17) using simple QP.

3. Calculate the corresponding $y_{\text {optimize }}^{1}(t+k \mid t)$

with eq. (13) and compare it to $y_{\text {base }}^{1}(t+k \mid t)$, which is the result of $u_{\text {base }}^{1}(t+k \mid t)$

4.1 In case $y_{\text {optimize }}^{1}$ is not small enough compared to $y_{\text {base }}^{1}: \quad$ re-define $\quad u_{\text {base }}(t+k \mid t) \quad$ as $u_{\text {base }}^{2}(t+k \mid t)=u_{\text {base }}^{1}(t+k \mid t)+\delta u^{1}(t+k / t)$ and go to step 2 . The underlying idea is that $u_{\text {base }}^{1}(t+k \mid t)+\delta u^{1}(t+k / t)$ which is the optimal $u(t+k \mid t)$ for a linear system - is an improved estimate for the optimal $u(t+k \mid t)$ in case of a nonlinear system

4.2 In case $y_{\text {optimize }}^{\mathrm{i}}(t+k \mid t)$ is small enough compared to $y_{\text {base }}^{i}(t+k \mid t)$ : use $u(t)=u_{\text {base }}^{i}(t \mid t)+\delta u^{i}(t / t)$ as the resulting control action of the current sampling instant $(i=1,2, \ldots$, according to the number of iterations).

This algorithm results after convergence to the optimal solution for the underlying nonlinear predictive control problem. The number of required iterations depends on how far the optimal $u^{*}(t+k / t)$ is away from the optimal $u^{*}(t+k / t-1)$. In case no big setpoint changes and no big disturbances occur between time instants $t$ - 1 and $t$, this number of iterations seems to be very low (1...3). Notice that a correct calculation of the local step response coefficients along the whole prediction horizon is not critical: these coefficients are only used to calculate $y_{\text {optimize }}(t+k \mid t)$, which is driven to zero anyhow.

\section{SIMULATION RESULTS}

Several simulation tests have been carried out to compare the standard NMPC with the non-linear EPSAC from the point of view of the computation time as well as the efficiency.

The sample period is $5 \mathrm{~min}$ whereas the other parameters are $N_{2}=\{15,15\}, N_{u}=\{1,1\}, \gamma=\{5,1\}, \beta=\{0,0\}$.

For the manipulated variables, the constraints were fixed to $\mathrm{u}_{\min }=\{2000,7000\}, \mathrm{u}_{\max }=\{5000,13000\}$ and their changes were limited to $\Delta \mathrm{u}_{\min }=\{-150,-1500\} ; \Delta \mathrm{u}_{\max }=\{150,1500\}$.

The controlled variables are constrained by $\mathrm{y}_{\min }=\{0,0\}$ and $\mathrm{y}_{\max }=\{0.05,0.4\}$.

In these simulations it is assumed that the full state measurement is available at time $t_{k}$, i.e. the initial condition is known at each iteration.

\section{Setpoint tracking}

During a simulation time of 4.5 hours, several step changes have been considered for both controlled variables. The performance obtained by both controllers was similar. For shortage of available space only results of one experiment are presented. Fig. 3 shows how the controller tries to make the molar concentration of ethanol at the bottom of the column to track the setpoint change from 0.0225 to 0.0246 at $t=0.2$ hours. The response of the second controlled variable, the molar concentration of the water at the top of the column, to the change of its reference from 0.1809 to 0.1654 at $t=1.8$ hours, is represented in Fig. 4. The two manipulated variables are represented in Figs. 5 and 6.

But the associated computational efforts indicate a clear advantage of the EPSAC controller. The computation time of the whole simulated experiment was almost 12 hours, whereas the standard nonlinear controller has taken approximately 53 hours. The simulation has been performed using the simulation language EcosimPro in a PC PentimIII, $800 \mathrm{MHz}$ computer with 512 Mbytes of RAM. 


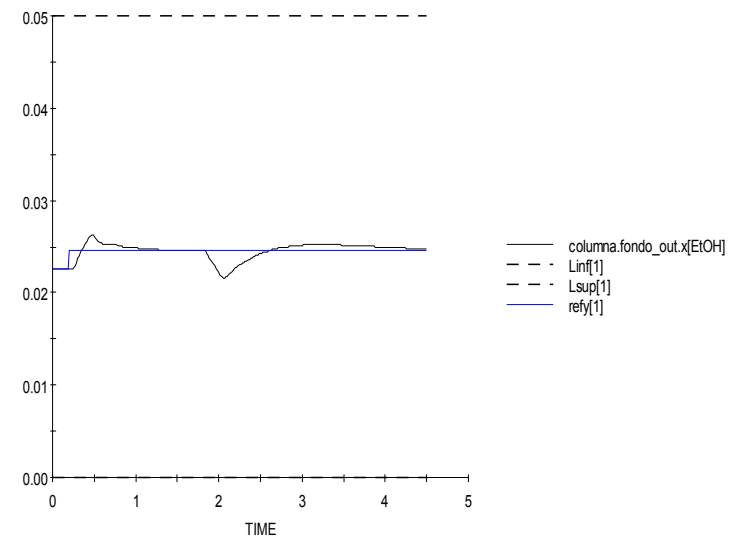

Fig. 3 Set point tracking of the first controlled variable (molar concentration of ethanol at the bottom of the column)

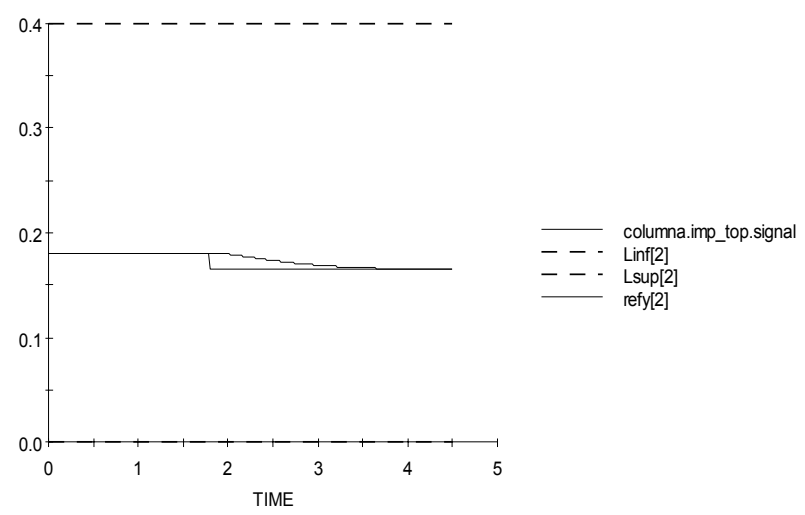

Fig. 4 Set point tracking of the $2^{\text {nd }}$ controlled variable (molar concentration of water at the top of the column)

\section{CONCLUSIONS}

Two non-linear model predictive controllers have been compared in a distillation column. One of them the nonlinear EPSAC, based on an iterative linearisation approach, has shown to be a promising technique in reducing computation time, cutting it to less than a fourth. However, in the process considered here, the time required to solve the predictive control problem every sampling time is still too high to implement the controller in real time.

The proposed method does not require to linearize the non-linear model, but only to compute its impulse response, which can save a lot of computation in cases like the one presented here.

Another advantage of the non-linear EPSAC refers to the use of codes more efficient such as QP instead of SQP methods. Further efforts are required to bring NMPC into practice when based on complex models like the one presented in this paper.

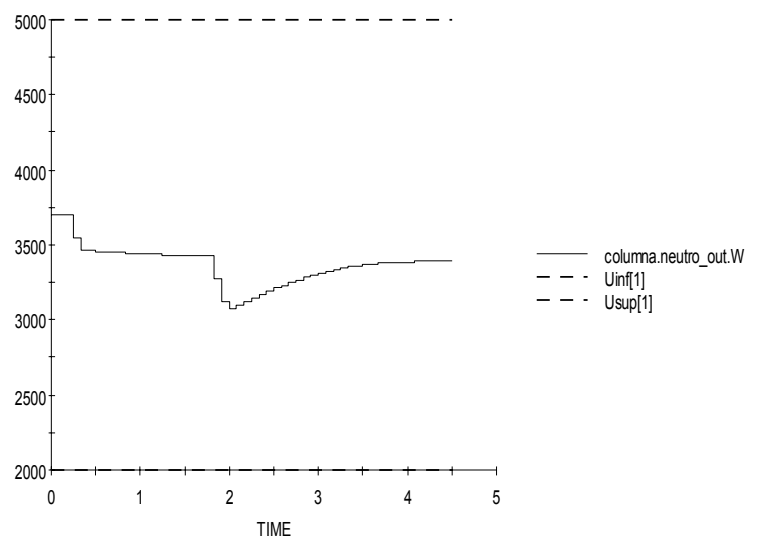

Fig. 5 The $1^{\text {st }}$ manipulated variable (neutral alcohol flow)

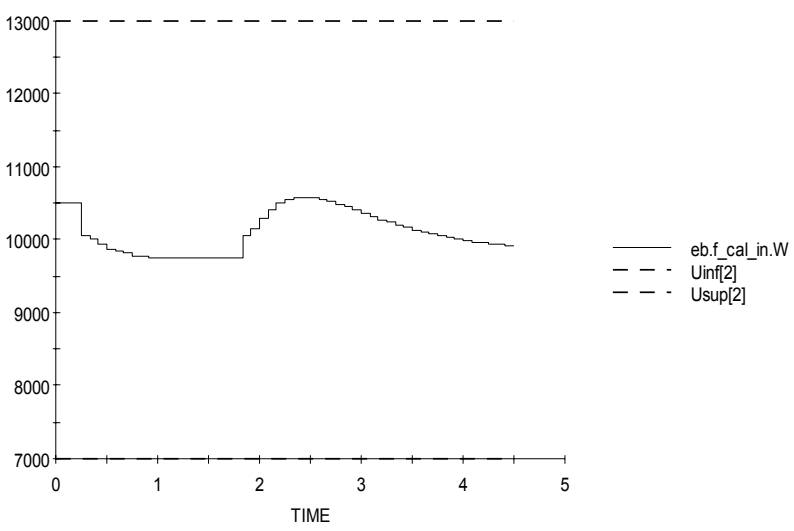

Fig. 6 The $2^{\text {nd }}$ manipulated variable (steam inflow to the reboiler)

\section{ACKNOWLEDGEMENTS}

The authors wish to express their gratitude to the Spanish CICYT for its support through the projects TAP97-1144C02-01 and PPQ2000-1075-C02-01, as well as to Belgian FWO-ICCOS and the Socrates Programme of the EU.

\section{NOMENCLATURE}

$M \quad$ Liquid mass accumulated on plate $(\mathrm{kg})$.

$L \quad$ Liquid flow $(\mathrm{kg} / \mathrm{s})$.

$V \quad$ Vapour flow $(\mathrm{kg} / \mathrm{s})$.

$F \quad$ Feed flow $(\mathrm{kg} / \mathrm{s})$. 
$S \quad$ Lateral extraction $(\mathrm{kg} / \mathrm{s})$.

$x \quad$ Mol fraction in liquid phase.

$y \quad$ Mol fraction of in vapour phase.

$z \quad$ Mol fraction in feed current .

$h \quad$ Enthalpy of the liquid $(\mathrm{kJ} / \mathrm{kg})$.

$H \quad$ Enthalpy of the $(\mathrm{kJ} / \mathrm{kg})$.

$\gamma \quad$ Activity coefficient.

$P^{\text {sat }} \quad$ Vapour pressure (bar).

$P \quad$ Total pressure (bar)

$\Delta P \quad$ Pressure drop (bar)

$V_{0} \quad$ Flow of live steam in the reboiler $\left(\mathrm{m}^{3} / \mathrm{s}\right)$

$\mathrm{K} \quad$ Constant of proportionality ( $\mathrm{m}^{3} /$ bar $\cdot \mathrm{s}$ )

$\mathrm{h}_{\mathrm{ow}} \quad$ Height of the liquid above the crest of the sink (mm)

Q Liquid which falls from the $\operatorname{sink}\left(\mathrm{m}^{3} / \mathrm{s}\right)$

$\mathrm{L}_{\mathrm{w}} \quad$ Length of the sink (m)

\section{Subscript}

j species

n stage

* molar fraction of vapour phase in equilibrium with $x_{n}^{j}$

\section{REFERENCES}

Bequette B.W. (1991) Nonlinear Control of Chemical Processes: A Review. Ind. Eng. Chem. Res., 30, 13911413.

E.F.Camacho and C.Bordons (2005) Nonlinear Model Predictive Control: an Introductory Survey. International Workshop on Assessment and Future Directions of Nonlinear Model Predictive Control. FreudenstadtLauterbad, Germany

De Keyser, R.M.C. (1998) A gentle introduction to model based predictive control. EC-PADI2 International Conference on Control Engineering and Signal Processing. Lima, Perú, Plenary Paper. Updated version(2003) published in "UNESCO Encyclopaedia of Life Support Systems", Article contribution 6.43.16.1, EoLSS Publishers Co Ltd, Oxford, ISBN 09542989 1826-34

Henson M.A. (1998) Nonlinear model predictive control: current status and future directions. Computers and Chemical Engineering, 23, 187-202.

H.G.Bocks. (2005). Numerical Methods for E_cient Nonlinear Predictive Control and Moving Horizon State Estimation. International Workshop on Assessment and Future. Directions of Nonlinear Model Predictive Control. Freudenstadt-Lauterbad, Germany

Kouvaritakis B. and Cannon M. (Editors). Nonlinear predictive control, theory and practice. IEE Control Engineering Series 61 (2001). The Institution of Electrical Engineers, London, U.K.
Morari M. and J. H. Lee (1999). Model predictive control: past, present and future. Computers and Chemical Engineering, 23, 667-682.

Rashid M. Ansari and Moses O. Tadé (2000). Nonlinear model based process control. Springer-Verlag.

R.D.Bartusiak (2005). NLMPC: A Platform for Optimal control of Feed- or Product-exible Manufacturing. International Workshop on Assessment and Future. Directions of Nonlinear Model Predictive Control. Freudenstadt-Lauterbad, Germany 